\title{
Improved Biological Tissue Preparation Procedure for Scanning Electron Microscopic Imaging
}

\author{
Habeeb Alsudani ${ }^{1,2}$, Soumitra Ghoshroy ${ }^{1,2}$, Joseph Quattro ${ }^{1}$, Matthew Greenwold ${ }^{1}$ and Roger Sawyer ${ }^{1}$ \\ 1. Department of Biological Sciences, University of South Carolina, Columbia, SC, USA \\ 2. Electron Microscopy Center, University of South Carolina, Columbia, SC, USA
}

Scanning Electron Microscope (SEM) is widely used to investigate surface architecture of a variety of specimens. Observation of most biological specimens under the SEM needs tissue preparation which is fairly well established [1]. Light microscopic observation of sectioned specimens that are paraffin embedded has its limitations. Low-vacuum scanning electron microscopy (SEM) has been used to observe biological samples by avoiding the "charging" impacts even in non-coated samples [2] with the occasional compromise of resolution. In the current study, the conventional protocol of biological sample preparation has been modified by merging a light microscope preparation protocol with that for SEM. This enables evaluation of internal structures of biological specimens by using SEM imaging technique.

Paraffin embedded biological specimens were sectioned using a Microm rotary microtome at a thickness range of $10-60 \mu \mathrm{M}$ and the ribbon was collected on an albumin coated thin aluminum support/plate, carbon sticky tab, and superfrost plus glass slide. The paraffin from the sections was removed using xylene, dried with Hexamethyldisilazane (HMDS) and all three substrates containing sections were mounted on a standard aluminum stub mount with the sections side facing up (fig. 1). The sectioned specimens were either gold coated with a Denton Desk II sputter coater and observed under a Tescan Vega 3 SEM outfitted with a secondary detector (SE) under high vacuum, or viewed uncoated using low vacuum SEM mode and a backscattered electron detector.

A variety of biological specimens that were loaded onto a thin aluminum plate, gold coated and observed under high vacuum mode with a SE detector, showed no "charging" effect and structural details of the specimens such as blood cells and epithelial tissue was clearly visible (fig. 3a, and 4a,b,c). The sections that were loaded onto sticky carbon tabs (fig. 3b,) or glass slide (fig. 3c), gold coated and imaged under high vacuum with SE detector showed considerable charging effects. Low vacuum environmental SEM imaging with different magnifications for non-gold coated specimens demonstrated a high variation in the image quality between aluminum plates (fig. 2a and fig.5a), sticky carbon tabs (fig. $2 \mathrm{~b}$ and fig. 5b), and glass slide (fig. 5c).

This study demonstrated that paraffin embedded sectioned tissues from various organisms loaded on a thin aluminum plate, coated with gold, and observed under high-vacuum with varying magnifications provided highest quality images when compared with sectioned specimens loaded on carbon tabs or glass slides. The gold coated sections fixed on albumin coated aluminum plates also stored well for several months in glass desiccators. This improved method eliminates the need for a variable pressure SEM for imaging paraffin embedded specimens and therefore would be highly effective for structural analysis in many areas of biology including histology, pathology, and embryology.

References:

[1] Ratnayake K, Joyce D and Webb R, Scientia Horticulturae 140 (2012), p. 12.

[2] Tanaka K, Inaga S and Lino A, J. Electron Microscopy 45 (1996), p. 344. 

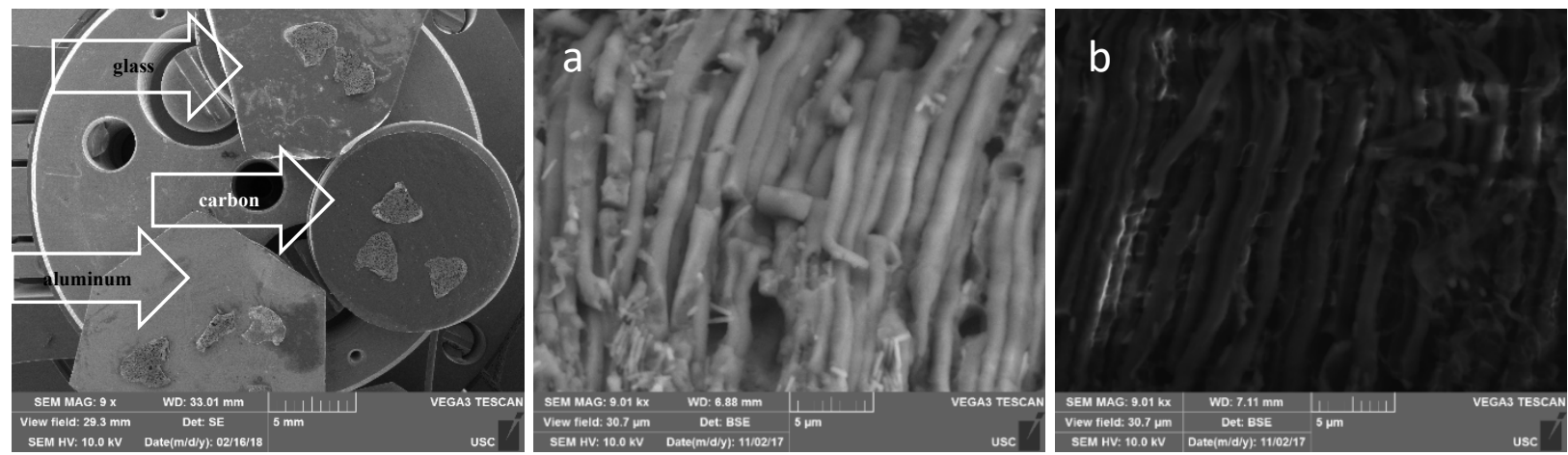

Figure 1. Image of SEM stage

Figure 2. SEM images of the uncoated retina of fish eyes in low-

with three mounting substrates vacuum loaded on aluminum plate (a) and carbon sticky tab (b)
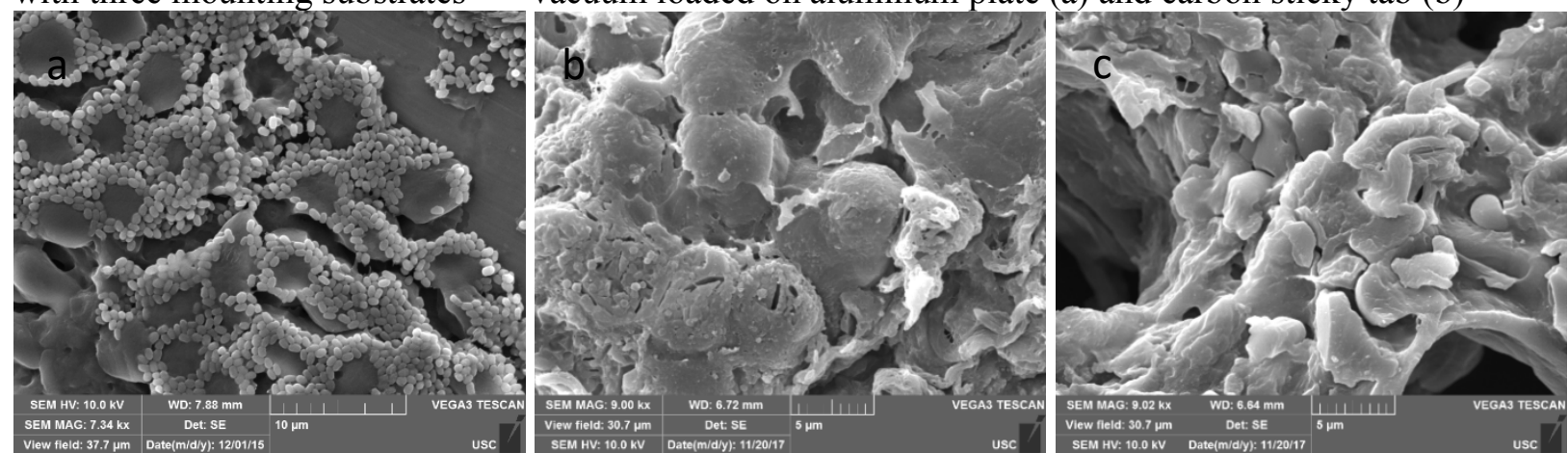

Figure 3. SEM images of mice lung coated with gold in high-vacuum, loaded on aluminum plate (a), sticky carbon tab (b), and glass slide (c).
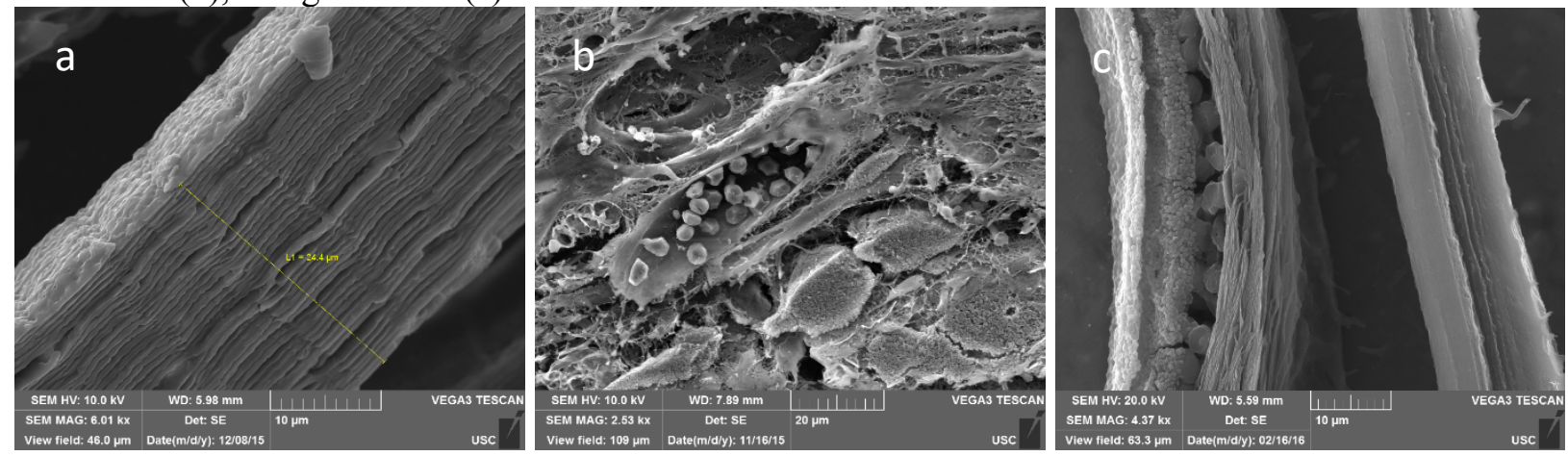

Figure 4. SEM images of fish eye lens (a), fish skin (b) and fish eye iris (c) tissues coated with gold in high-vacuum, loaded on an aluminum plate with varying magnifications.
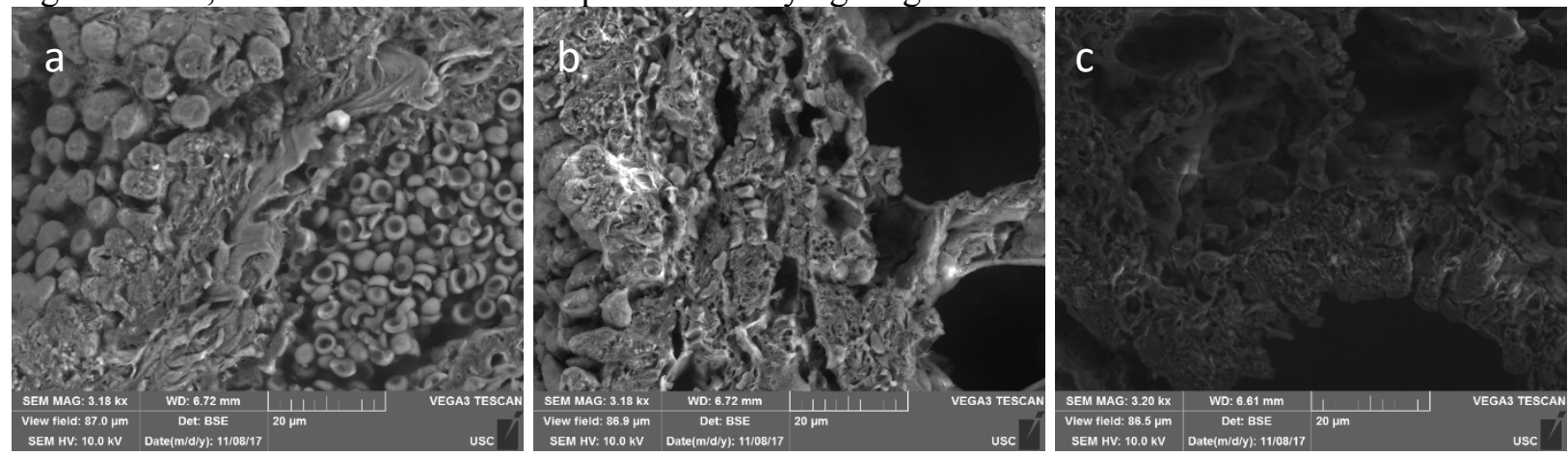

Figure 5. SEM images of uncoated mouse lung in low-vacuum, loaded on aluminum plate (a), carbon sticky tab (b), and glass slide (c). 